\title{
Decay Process Characteristics and Fungal Community Composition of Salix psammophila Sand Barriers in an Arid Area, Northern China
}

\author{
Xia Yang, Yumei Liang, Yong Gao * and Ruidong Wang *
}

Citation: Yang, X.; Liang, Y.; Gao, Y.; Wang, R. Decay Process

Characteristics and Fungal

Community Composition of Salix psammophila Sand Barriers in an Arid Area, Northern China. Sustainability 2021, 13, 7590. https://doi.org/ $10.3390 /$ su13147590

Academic Editor: Sean Clark

Received: 18 May 2021

Accepted: 5 July 2021

Published: 7 July 2021

Publisher's Note: MDPI stays neutral with regard to jurisdictional claims in published maps and institutional affiliations.

Copyright: (c) 2021 by the authors. Licensee MDPI, Basel, Switzerland. This article is an open access article distributed under the terms and conditions of the Creative Commons Attribution (CC BY) license (https:// creativecommons.org/licenses/by/ $4.0 /)$.
Key Laboratory of Eolian Physics and Desertification Control Engineering, College of Desert Control Science and Engineering, Inner Mongolia Agricultural University, 29 Erdos East Street, Hohhot 010018, China; yangxiashamoyuan@imau.edu.cn (X.Y.); liang490002878@163.com (Y.L.)

* Correspondence: 13948815709@163.com (Y.G.); wangruidong0226@163.com (R.W.)

\begin{abstract}
With the increase in setting years in deserts, Salix psammophila sand barriers with different degrees of lodging damage caused by decay are losing wind-prevention and sand-fixation properties. In this study, we focus on the change in chemical properties of soils, and physical and mechanical properties of plants along different setting years; meanwhile, the change in fungal communities has been analyzed using high-throughput sequencing technology. The results show that a change in physical and mechanical properties and the loss of primary chemical components led to the degradation of the protective properties of the barrier to different degrees. After five years of setting, the physical parameters of basic density and shrinkage rate decreased by $44.04 \%$ and $28.68 \%$, respectively, and the loss of the modulus of rupture mechanical index declined by $62.72 \%$. After seven years of setting, the mechanical indexes of the modulus of rupture decreased by $76.95 \%$. Five and seven years represented important inflection points in the decay process. Sordariomycetes (53.75\%) and Eurotiomycetes (19.78\%) were the main fungal groups present during the decay of the sand barrier. The basic density, moisture content, cellulose, and lignin of the sand barrier were the main driving factors affecting the distribution of fungal communities. The mechanism on fungal community to the decay of sand barriers still needs further studies to keep the function of sand barriers in fragile desert ecosystems.
\end{abstract}

Keywords: S. psammophila sandbarrier; wind-preventing and sand-fixing effects; decay process; fungal community diversity

\section{Introduction}

The use of shrub branches as sand barriers to prevent sand damage, control desertification, and improve the fragile ecological environment of desert ecosystems has become an increasingly relevant field of research [1]. Salix psammophila (C. Wang et Ch. Y. Yang) is a typical desert shrub with drought-resistant characteristics and strong growth capacity that can withstand wind erosion, sand burial, and other harsh environmental conditions. It is mainly distributed in mobile and semi-mobile dunes as well as interdune lowlands in northwest China [2,3]. As sand barriers offer the dual advantages of engineering sand control and biological sand control, sand barriers of different specifications are placed on moving dunes after leveling the stubble [1]. Sand barriers consisting of S. psammophila are one of the main measures for sand control, as they have good wind-prevention and sand-fixation properties, improve the properties of the soil and water, and are suitable for vegetation restoration $[4,5]$. As the duration from the setting of $S$. psammophila sand barriers increases, the sand barriers experience significant impacts from natural factors, such as changes in the temperature and humidity of the environment, microbial erosion, and so forth. If the sand barrier is present in a sandy soil environment for a long time it will decay, leading to significant damage and collapse of the sand barrier. Studies show that 
the modulus of rupture (MOR) of $S$. psammophila sand barriers decreases and the lodging damage rate increases at five years from setting, during which the damage rate of the sand barrier can reach $80 \%$, thus seriously hampering the wind and sand protection benefits of the barrier [4].

Among natural factors, microbial activity is a key factor affecting the degradation of the structure and the properties of natural biomaterials. Fungi are stress-tolerant eukaryotic organisms. They can grow in the absence of water and resist drought by forming spores and other structures, which have the most direct influence on the durability of biological materials [6,7]. It has been reported that saprophytic fungi are the main group that lead to the decay of natural biomaterials [8] and are able to grow and reproduce rapidly in a suitable environment. At the same time, saprophytic fungi produce a variety of enzymes that decompose cellulose and lignin, which accelerates the decay rate of S. psammophila sand barriers and plays an important role in material circulation and energy conversion in desert ecosystems [9]. Changes in the internal microenvironment during the decay process of $S$. psammophila sand barriers affect the growth and species composition of the fungal community [10-12]. Studying the physiological and ecological functions of the fungal community, the relationship between saprophytic fungi and desert ecological environment, and the evolution of the fungal community during the decay process of $S$. psammophila sand barriers is thus significant. However, studies on S. psammophila sand barriers in desert areas have mostly focused on ecological protection benefits [2,3], and there is limited information on fungal community changes and characteristics during the process of sand barrier decay. Additionally, compared with other ecological environments, studies on saprophytic fungal communities in extreme arid environments have not been reported.

In the present study, we assumed that the degradation of $S$. psammophila sand barriers may be related to a change in chemical composition and the loss of physical and mechanical properties, and that an inflection point could exist along the setting years. At the same time, a biological process could occur, which could be caused by a change in the fungal community. To test these hypotheses, we combined the assessment of the physical and mechanical properties, chemical composition for plants and soils, and high-throughput sequencing technology for fungal communities along different setting years. The results could lay a foundation for future studies on the interaction of sand barriers and fungal communities.

\section{Materials and Methodology}

\subsection{Materials}

The study was conducted at the Hangjin Banner, Ordos, Inner Mongolia in arid northwestern China $\left(108^{\circ} 41^{\prime} 21^{\prime \prime}\right.$ E, $\left.40^{\circ} 29^{\prime} 34^{\prime \prime} \mathrm{N}\right)$. Salix psammophila sand barriers with specifications of $1 \mathrm{~m} \times 1 \mathrm{~m}$ that had been setting for one, three, five, seven, and nine years on gentle dunes were selected for investigation and sampling (Figure 1). Sampling consisted of two parts: plant material sample of S. psammophila sand barrier (sand barrier samples) and surrounding soil sample of S. psammophila sand barrier.

Sand barrier sample: three representative sampling sites were selected from each sample site, and six samples were taken from each sample site. Samples from the east, south, west, and north directions of the sand barrier with a diameter of $1.8 \pm 0.02 \mathrm{~cm}$ and a depth of $20 \pm 0.5 \mathrm{~cm}$ were collected as test samples from the sand-buried part of the sand barrier at each sample. A total of 90 sand barrier samples were selected as the sand barrier sample. The sand barrier samples with a length of $7 \mathrm{~cm}$ at the bottom of the sand barrier were cut off and taken for the determination of the physical and mechanical properties and chemical components of the S. psammophila sand barriers. DNA extraction of S. psammophila sand barrier fungus: a sterilized brush was used to clean off the sand barrier soil. The sand barrier samples with a length of $7 \mathrm{~cm}$ at the bottom of the sand barrier were cut off and placed into a sterile sealed bag and marked, stored in an ice box, and shipped back to the laboratory immediately. The samples were crushed on an ultra-clean table, and the four samples from the same barrier were mixed evenly into one sample and placed into a $15 \mathrm{~mL}$ CORNING CentriStar ${ }^{\mathrm{TM}}$ sterile centrifuge tube. With three replicates for each group, a 
total of 60 samples were sampled. The samples were stored at $-8^{\circ} \mathrm{C}$ for DNA extraction of S. psammophila sand barrier fungus.
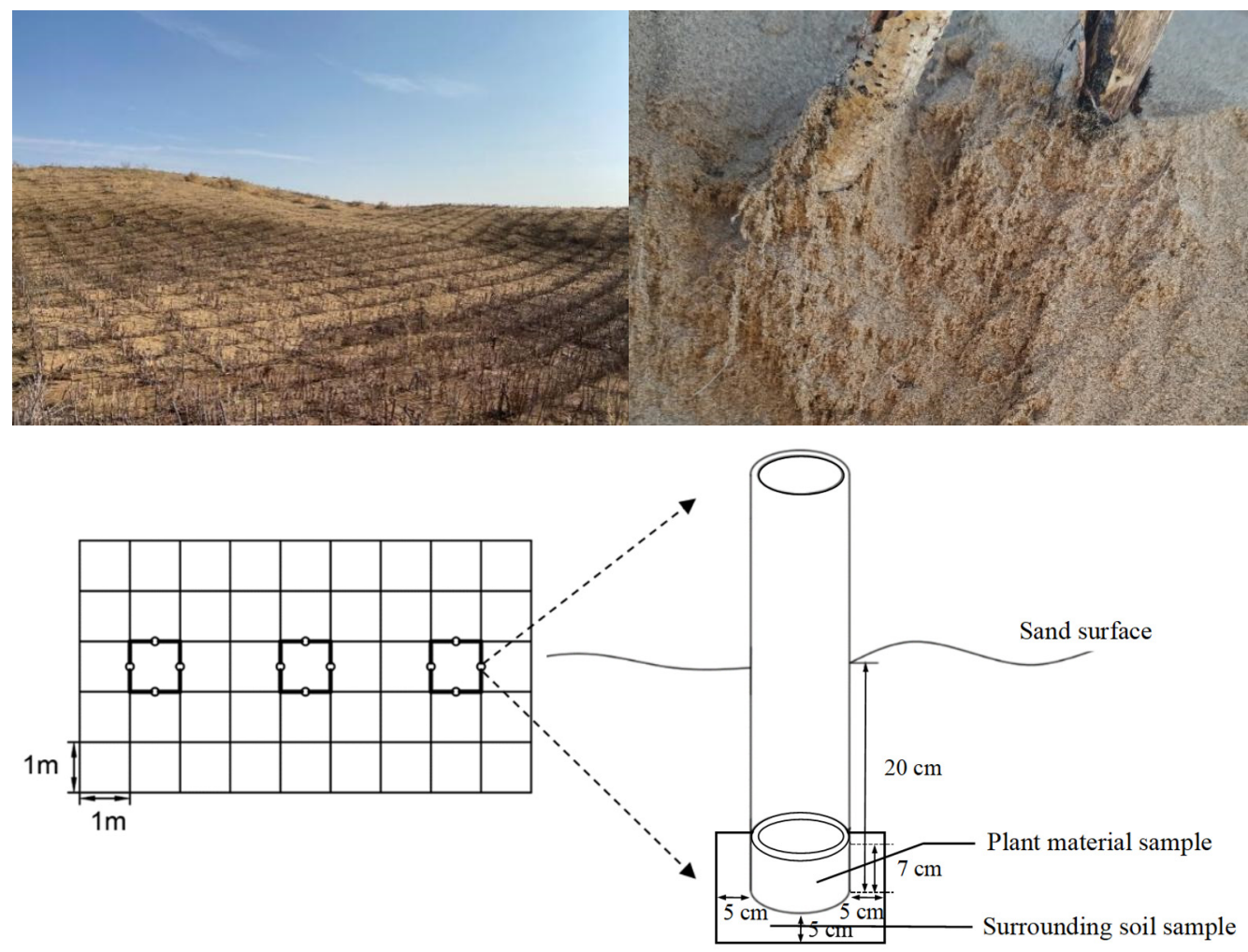

Figure 1. Schematic diagram of Salix psammophila sand barrier sample site.

Surrounding soil sample of S. psammophila sand barrier: the collection of the surrounding soil corresponded to the collection of $S$. psammophila sand barrier plant materials with a depth of $20 \pm 0.5 \mathrm{~cm}$, including the soil with a vertical length of $7 \mathrm{~cm}$ and a horizontal width of $5 \mathrm{~cm}$. The surrounding soil corresponding to the buried part of the $S$. psammophila sand barrier was collected, with three replicates for each group; a total of 60 samples were sampled. The samples were placed into plastic bags and taken back to the laboratory to remove impurities. The four soil samples from the same sample site were mixed evenly and made into one composite sample, which was mainly used for the determination of the physical and chemical properties of the soil.

\subsection{Methodology}

2.2.1. Determination of the Physical-Mechanical Properties and Chemical Analysis of the S. Psammophila Sand Barrier

The sand barrier samples were placed in a manual climatic box at a temperature of $20 \pm 2{ }^{\circ} \mathrm{C}$ and a relative humidity of $65 \pm 5 \%$, according to the National Forestry Industry Standard LY/T 2369-2014 “Test Method for Physical and Mechanical Properties of Sammophytic Shrubs" [13]. The moisture content of the sand barrier samples was adjusted to meet the national standard mechanical test requirements of 9-15\% moisture content for physical and mechanical index testing. The mass was weighed using an electronic balance at $0.001 \mathrm{~g}$ precision. The diameter and length of the sand barrier samples were measured with an electronic digital display caliper, and their physical properties were calculated. The physical properties such as the basic density, dry-shrinkage rate, and moisture content, as well as the mechanical properties of the MOR, were determined using a universal testing machine (Model TY-8000, Tianyuan, Jiangsu, China).

The chemical composition of the $S$. psammophila sand barrier was determined. Wood powders that were passed through a 40-60-mesh sieve were dried at $80^{\circ} \mathrm{C}$ to a constant weight. Cellulose was determined by the nitric acid-ethanol method, hemicellulose was de- 
termined by the hydrochloric acid-DNS reducing sugar method, and lignin was determined by the concentrated sulfuric acid solution method with $72 \%$ concentration [14-16].

\subsubsection{Determination of the Physical and Chemical Properties of the Surrounding Soils}

Soil moisture content was measured using the drying method and soil $\mathrm{pH}$ was measured by a pH meter. Alkali-hydrolyzable nitrogen (N) was determined by the alkalihydrolyzable diffusion method. Soil-available phosphorus $(\mathrm{P})$ and potassium $(\mathrm{K})$ were determined by the $0.5 \mathrm{~mol} / \mathrm{L} \mathrm{NaHCO}_{3}$ method and $\mathrm{NH}_{4} \mathrm{OA}_{C}$-flame spectrophotometry. Soil total $\mathrm{P}$ and total $\mathrm{K}$ were determined by the perchloric acid-sulfuric acid method and sodium hydroxide fusion-flame photometric method, respectively [17].

\subsubsection{DNA Extraction and Illumina MiSeq Sequencing}

The fungal communities were profiled by Illumina amplicon sequencing. Total genomic DNA was extracted from $0.5 \mathrm{~g}$ of each subplot sample using a Fast DNA ${ }^{\circledR}$ SPIN Kit (MP Biomedicals, Solon, OH, USA), following the instructions. After pooling the DNA at the plot level, the internal transcribed spacer (ITS) region, a widely used fungal marker [18,19], was targeted for DNA amplification. For the PCR, we used the universal primers ITS1F and ITS2R. Amplification of each sample was performed in triplicate using $0.4 \mu \mathrm{L}$ of TransStart ${ }^{\circledR}$ FastPfu DNA Polymerase (TransGen AP221-02) and $0.8 \mu \mathrm{L}(5 \mu \mathrm{m})$ of each primer with template DNA (10 $\mathrm{ng} / \mu \mathrm{L}$ concentration to amplify) with a final volume of $20 \mu \mathrm{L}$. The PCR conditions were as follows: $3 \mathrm{~min}$ at $95^{\circ} \mathrm{C}$, followed by 35 cycles of $95{ }^{\circ} \mathrm{C}$ for $30 \mathrm{~s}, 55^{\circ} \mathrm{C}$ for $30 \mathrm{~s}$, and $72{ }^{\circ} \mathrm{C}$ for $45 \mathrm{~s}$, with a final extension of $72{ }^{\circ} \mathrm{C}$ for $10 \mathrm{~min}$. The PCR product was extracted from $2 \%$ agarose gel and purified using the AxyPrep DNA Gel Extraction Kit (Axygen Biosciences, Union City, CA, USA) and quantified using a Quantus $^{\mathrm{TM}}$ Fluorometer (Promega, Madison, WI, USA). Purified amplicons were pooled in equimolar ratios and paired-end sequenced $(2 \times 300)$ on an Illumina MiSeq platform (Illumina, San Diego, CA, USA) according to the standard protocols by Majorbio Bio-Pharm Technology Co. Ltd. (Shanghai, China).

\subsubsection{Bioinformatics}

Raw fungal sequences of each sample were extracted based on barcodes, quality filtered by Trimmomatic, and merged by FLASH. The 300-bp reads were truncated at any site receiving an average quality score of $<20$ over a 50 -bp sliding window, and the truncated reads shorter than $50 \mathrm{bp}$ were discarded, as were reads containing ambiguous characters. Only overlapping sequences longer than $10 \mathrm{bp}$ were assembled according to their overlapping sequence. The maximum mismatch ratio of overlap region was 0.2 . Reads that could not be assembled were discarded. The sequences were clustered into operational taxonomic units (OTUs) by $97 \%$ sequence identity using UPARSE (version 7.1, http:/ / drive5.com/uparse/ (accessed on 30 January 2020)). The taxonomy of the most abundant read per OTU was assigned according to the UNITE (Release 7.2 http:/ / unite. ut.ee/index.php (accessed on 30 January 2020)) reference database using the Bayesian classifier [18].

\subsection{Data Analysis}

According to the results of the taxonomic identification, the community bar chart was drawn using R software (www.r-project.org (accessed on 30 January 2020)) to reflect the species composition and relative abundance at a certain taxonomic level. The alpha diversity index of the fungal community at different sequencing depths was calculated using Mothur software. The $\mathrm{R}$ vegan package was used to draw the redundancy analysis (RDA) diagram. One-way ANOVA was used to statistically analyze the differences between various indexes of sand barrier samples and surrounding soil samples as well as alpha diversity indices. Under the assumption of statistical normality and homogeneity of variance, the one-sample t-test was conducted to determine significance, which was subject to normal distribution. Under the effect of significant interaction, the least significant 
difference comparison was used for post-facto comparison, and the statistical analysis software SPSS 22.0 (IBM Corp., Armonk, NY, USA) was used for data analysis.

\section{Results}

\subsection{Characteristics of the Surrounding Soil Moisture and Nutrient Changes}

As shown in Table 1, the soil moisture content surrounding the S. psammophila sand barrier showed an overall downward trend with the increase in setting years. After setting for one year, the soil moisture content decreased rapidly. Setting for three to seven years resulted in the soil moisture content being basically maintained within a small range of fluctuations. After setting for seven years, the soil moisture content gradually decreased to the lowest figure at nine years of setting, $1.97 \%$. The $\mathrm{pH}$ of the surrounding soil during soil setting from one to seven years fluctuated and declined, with no significant differences observed $(p<0.05)$. Setting of seven to nine years exhibited an increasing trend, with a maximum value of 9.17, and was significantly different from the other years $(p<0.05)$. This illustrates that the protection of $S$. psammophila sand barriers decreased with time: soil moisture decreased, but soil $\mathrm{pH}$ increased.

Table 1. The characteristics of the surrounding soil moisture and nutrient changes.

\begin{tabular}{|c|c|c|c|c|c|c|c|}
\hline \multirow{2}{*}{$\begin{array}{c}\text { Different } \\
\text { Setting Years (y) }\end{array}$} & \multicolumn{7}{|c|}{ Plot Information } \\
\hline & $\begin{array}{c}\text { Soil Moisture } \\
(\%)\end{array}$ & Soil pH & $\begin{array}{c}\mathrm{AN} \\
\left(\mathrm{mg} \mathrm{kg}^{-1}\right)\end{array}$ & $\begin{array}{c}\mathrm{AP} \\
\left(\mathrm{mg} \mathrm{kg}^{-1}\right)\end{array}$ & $\begin{array}{c}\mathrm{AK} \\
\left(\mathrm{mg} \mathrm{kg}^{-1}\right)\end{array}$ & $\begin{array}{c}\text { TK } \\
\left(\mathrm{g} \mathrm{kg}^{-1}\right)\end{array}$ & $\begin{array}{c}\mathrm{TP} \\
\left(\mathrm{g} \mathrm{kg}^{-1}\right)\end{array}$ \\
\hline $1 \mathrm{y}$ & $3.36 \pm 0.36^{a}$ & $8.81 \pm 0.23^{b c}$ & $0.97 \pm 0.23^{b}$ & $0.30 \pm 0.03^{a}$ & $6.30 \pm 0.32^{\mathrm{ab}}$ & $0.28 \pm 0.01^{\mathrm{a}}$ & $16.59 \pm 1.17^{\mathrm{a}}$ \\
\hline $3 y$ & $2.59 \pm 0.17^{\mathrm{ab}}$ & $8.55 \pm 0.18^{b c}$ & $0.98 \pm 0.21^{b}$ & $0.25 \pm 0.05^{\mathrm{ab}}$ & $6.84 \pm 0.24^{\mathrm{a}}$ & $0.27 \pm 0.01^{\mathrm{a}}$ & $16.46 \pm 0.36^{\mathrm{a}}$ \\
\hline $5 y$ & $2.46 \pm 0.21^{\mathrm{ab}}$ & $8.79 \pm 0.17^{b c}$ & $1.02 \pm 0.26^{b}$ & $0.25 \pm 0.05^{\mathrm{ab}}$ & $6.27 \pm 0.27^{\mathrm{ab}}$ & $0.28 \pm 0.01^{\mathrm{a}}$ & $16.58 \pm 1.30^{\mathrm{ab}}$ \\
\hline $7 y$ & $2.51 \pm 0.45^{\mathrm{ab}}$ & $8.43 \pm 0.22^{c}$ & $1.22 \pm 0.10^{b}$ & $0.20 \pm 0.04^{\mathrm{ab}}$ & $6.43 \pm 0.12^{a b}$ & $0.27 \pm 0.02^{\mathrm{a}}$ & $14.79 \pm 0.40^{b}$ \\
\hline $9 y$ & $1.97 \pm 0.14^{\mathrm{b}}$ & $9.17 \pm 0.07^{\mathrm{a}}$ & $1.75 \pm 0.07^{\mathrm{a}}$ & $0.17 \pm 0.01^{\mathrm{b}}$ & $5.90 \pm 0.10^{b}$ & $0.25 \pm 0.03^{a}$ & $16.27 \pm 1.05^{\mathrm{ab}}$ \\
\hline
\end{tabular}

Note: Different lowercase letters in the figure indicate significant difference between different setting years $(p<0.05)$. AN, Soil alkalihydrolyzable nitrogen; $\mathrm{AP}$, available phosphorus; $\mathrm{AK}$, available potassium; TK, total potassium; and TP, total phosphorus.

Soil alkali-hydrolyzable $\mathrm{N}$ showed an increasing trend with the increase in setting years. The change in soil alkali-hydrolyzable $\mathrm{N}$ content was small after setting for one to three years, whereas its rate of increase was relatively rapid after setting for five years, reaching the maximum value of $1.75 \mathrm{mg} / \mathrm{kg}$ after nine years of setting, which was significantly higher than at one year of setting $(p<0.05)$. The change in available $\mathrm{P}$ is shown in Table 1. Contrary to the change in alkali-hydrolyzable $\mathrm{N}$ content, the available P content decreased rapidly after one year of setting and stabilized between three and five years of setting. After five years, the soil-available P decreased relatively rapidly. The content of soil-available K generally showed a decreasing trend with the increase in setting years. The contents of total $\mathrm{P}$ and total $\mathrm{K}$ in the surrounding soil of the S. psammophila sand barriers remained largely stable from one year to five years of setting. After seven years, the rate of decrease in total P content increased. The lowest total P content was $14.79 \mathrm{~g} / \mathrm{kg}$ after setting for seven years, which was significantly different from that of setting for one to three years $(p<0.05)$. The above results indicate that the $S$. psammophila sand barrier mainly affected the soil moisture, $\mathrm{pH}$, alkali-hydrolyzable $\mathrm{N}$, and available $\mathrm{P}$ in the surrounding soil during the entire effective protection period, but it had little effect on the content of total $\mathrm{P}$ and total $\mathrm{K}$ in the soil.

\subsection{The Decay Characteristics of the S. Psammophila Sand Barrier}

3.2.1. Physical and Mechanical Properties

With the increase in setting years, the density of the S. psammophila sand barrier showed a slow decreasing trend, while the sand barrier sample moisture content and dry-shrinking rate showed a slow increasing trend, all of which showed a linear function relationship with the setting year. The density of the sand barrier decreased by $26.23 \%$ from one year to three years. The dry-shrinkage rate showed a rising trend, but the moisture content of the sand barrier samples increased to about $67.9 \%$ after three years. The sand barriers underwent changes that increased their water absorption properties. With the increase in 
setting years, the size of the S. psammophila sand barrier exhibited an obvious shrinkage phenomenon. After setting for three to seven years, the density of the S. psammophila sand barrier changed gradually, and the dry-shrinkage rate increased significantly from $13.41 \%$ to $21.09 \%$. The moisture content of the sand barrier samples increased steadily. The basic density of the S. psammophila sand barrier decreased by $37.07 \%$ when the setting time exceeded seven years. The curves of the water content and dry-shrink year rate changed gently. After seven years of setting, the state of decay was relatively stable.

The MOR of a natural biomaterial is the most important mechanical property in its resource utilization [20]. As shown in Figure 2, with the increase in setting years, the MOR index showed a downward trend, and the decline was rapid at first and then slowed. After setting for seven years, the MOR decreased by $76.95 \%$. As the water content of the S. psammophila sand barrier increased in this interval, the dimensional stability of the sand barrier changed, as did the performance of the sand barrier, and thus the mechanical properties of the S. psammophila sand barrier decreased. When the setting years exceeded seven years, the downward trend in the MOR curve declined.
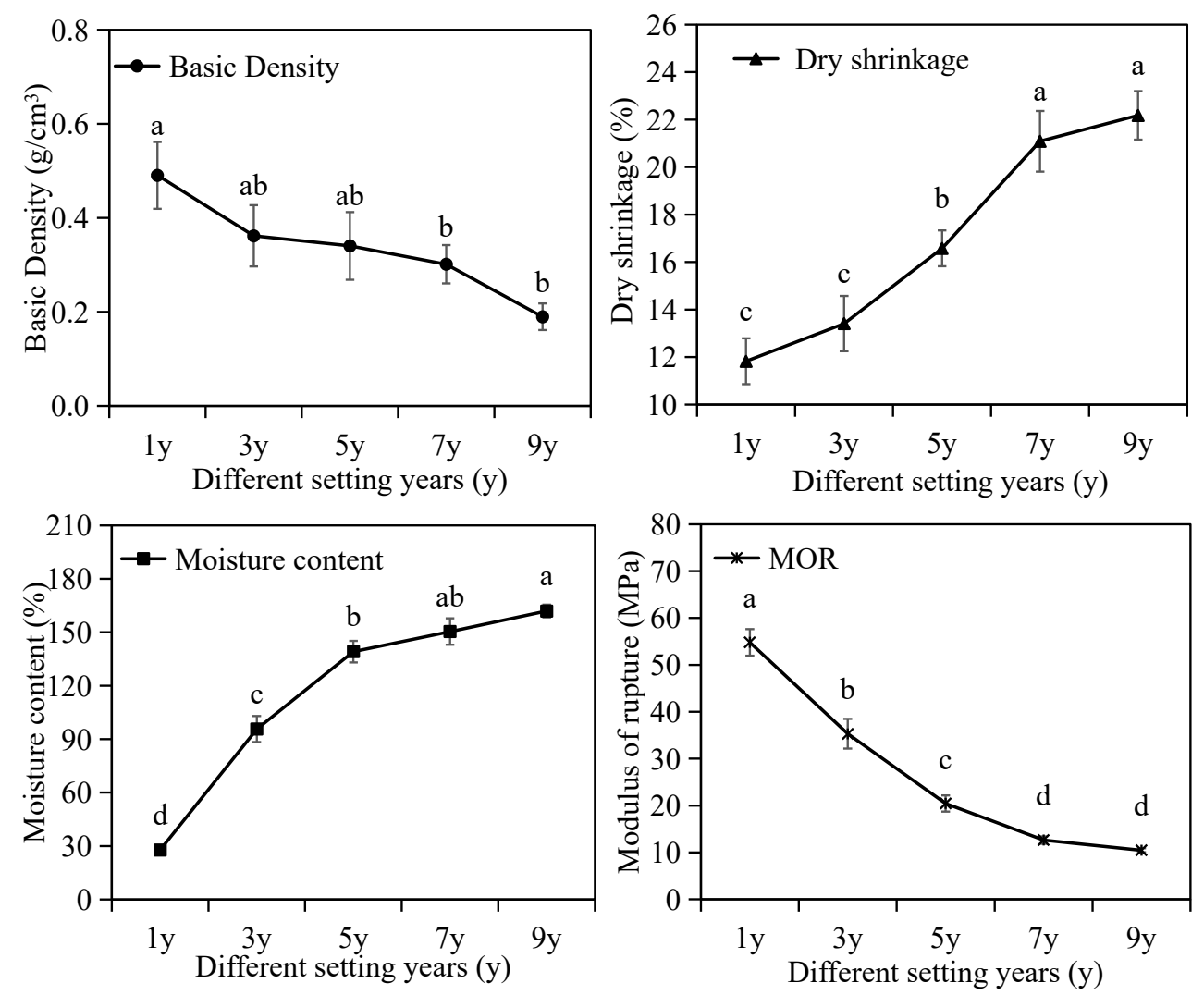

Figure 2. Physical and mechanical properties of the S. psammophila sand barrier. Note: different lowercase letters in the figure indicate significant differences between different setting years of the sand barrier $(p<0.05)$.

\subsubsection{Contents of the Main Chemical Components}

To elucidate the changes in the chemical composition of S. psammophila sand barriers after long-term use, quantitative analysis of the chemical composition of S. psammophila sand barriers was carried out. The sand barriers are mainly composed of cellulose (skeleton material, providing strength), hemicellulose (adhesive material, providing shear resistance), and lignin (filling material, providing hardness), which affect the ability of S. psammophila sand barriers to prevent wind and fix sand [21]. The contents of cellulose, hemicellulose, and lignin in the sand barriers showed a decreasing trend with the increase in setting years (Figure 3). According to variance analysis, there were significant differences in the content 
changes of the main chemical components of the S. psammophila sand barriers with different setting years, and there was a certain coordination relationship $(p<0.05)$.
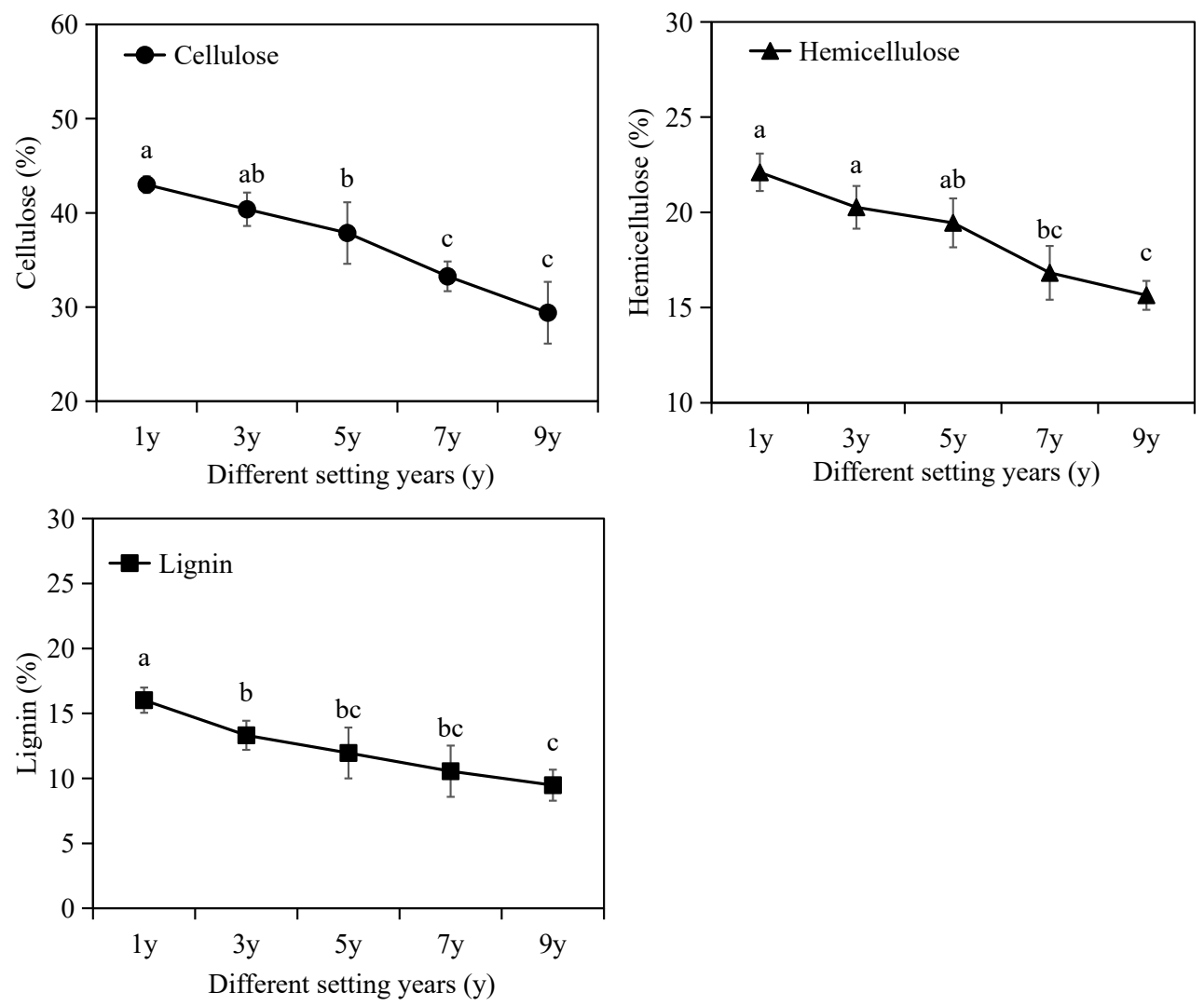

Figure 3. The main chemical components of the S. psammophila sand barrier. Note: different lowercase letters in the figure indicate significant differences between different setting years $(p<0.05)$.

The cellulose content, hemicellulose content, and lignin content of the S. psammophila sand barrier decreased after five years of setting by $9.4 \%, 5.46 \%$, and $4.93 \%$, respectively, compared with after one year of setting. The main chemical composition of the Salix sand barrier changed greatly between three and seven years after setting. After setting for nine years, the cellulose content decreased by $17.14 \%$, the hemicellulose content decreased by $9.06 \%$, and the lignin content decreased by $7.66 \%$ compared with one year. The content of the main chemical components of the S. psammophila sand barrier decreased with the increase in setting years.

\subsection{Species Composition and Diversity at the Fungal Level}

As shown in Figure 4, species information of 20 classes of fungi were annotated in the sand burial samples from different years. The average relative abundances of Sordariomycetes, Eurotiomycetes, Agaricomycetes, Leotiomycetes, c__unclassified_p_Ascomycota, Dothideomycetes, p_unclassified_k_Fungi and Mortierellomycetes were $53.75 \%, 19.78 \%, 11.65 \%, 7.21 \%, 5.60 \%$, $0.85 \%, 0.52 \%$, and $0.45 \%$, respectively. The sum of the relative abundances of species accounted for $99.81 \%$ of the total abundance. The species abundance ratio of Sordariomycetes in the samples set for one and three years was higher than that in the samples set for five, seven, and nine years, while the relative abundance ratio of Eurotiomycetes exhibited the opposite pattern. Leotiomycetes accounted for the largest proportion after five years of setting, with a relative abundance of $21.93 \%$, while Leotiomycetes accounted for the lowest proportion at one year and three years of setting, with a relative abundance of $0.01 \%$ and $0.03 \%$, respectively. 


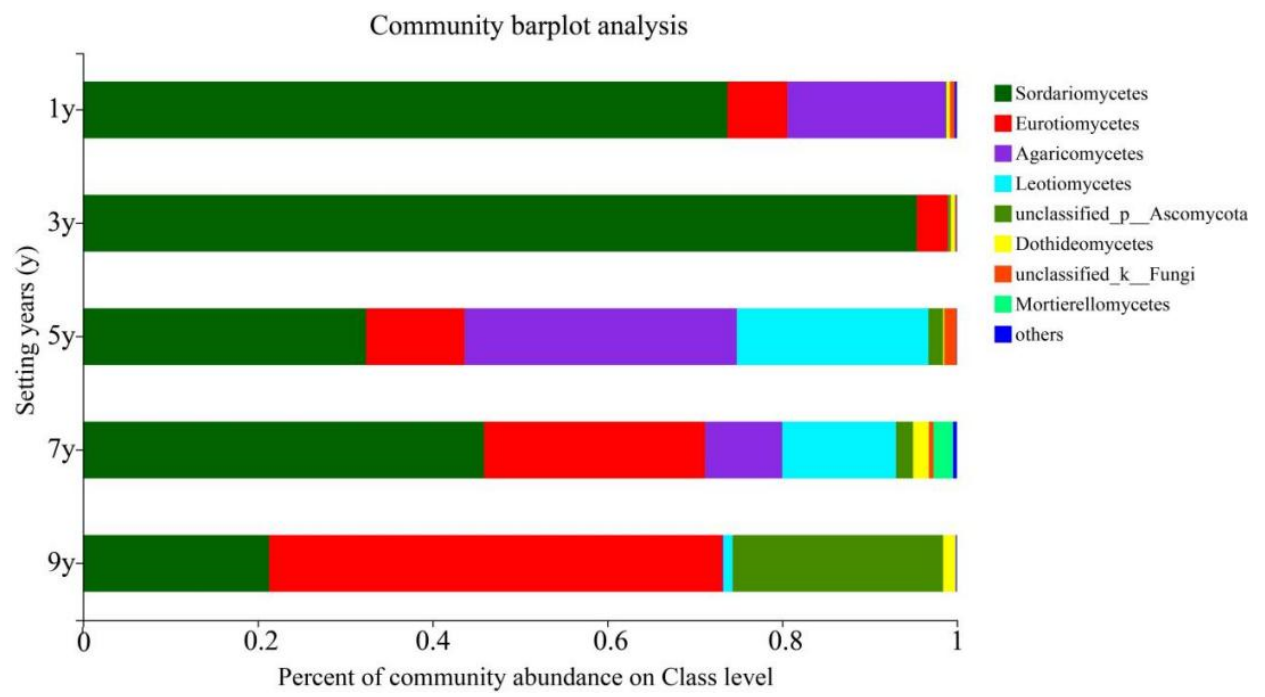

Figure 4. Relative abundance of species at the level of the fungal class in S. psammophila sand barriers in different years.

The Shannon index and Simpson index were used to reflect the diversity of the fungal community, while the Sobs index and Ace index were used to reflect the richness of the fungal community. The statistical results of the Alpha diversity index (as shown in Figure 5) showed that the Sobs and Ace indexes of the fungal community with different setting years showed a trend of first increasing and then decreasing with the increase in setting time. The Shannon index ranged from 0.19 to 1.27 , and there were significant differences in the Shannon index values among groups of different years $(p<0.05)$, whereas there were no significant differences in the diversity between one year of installation and three, five, and seven years of installation $(p>0.05)$. The Simpson index values ranged from 0.33 to 0.92 for different setting years of S. psammophila sand barriers, and the Simpson index of community dominant degree setting for three years was significantly different from that of five, seven, and nine years $(p<0.05)$.
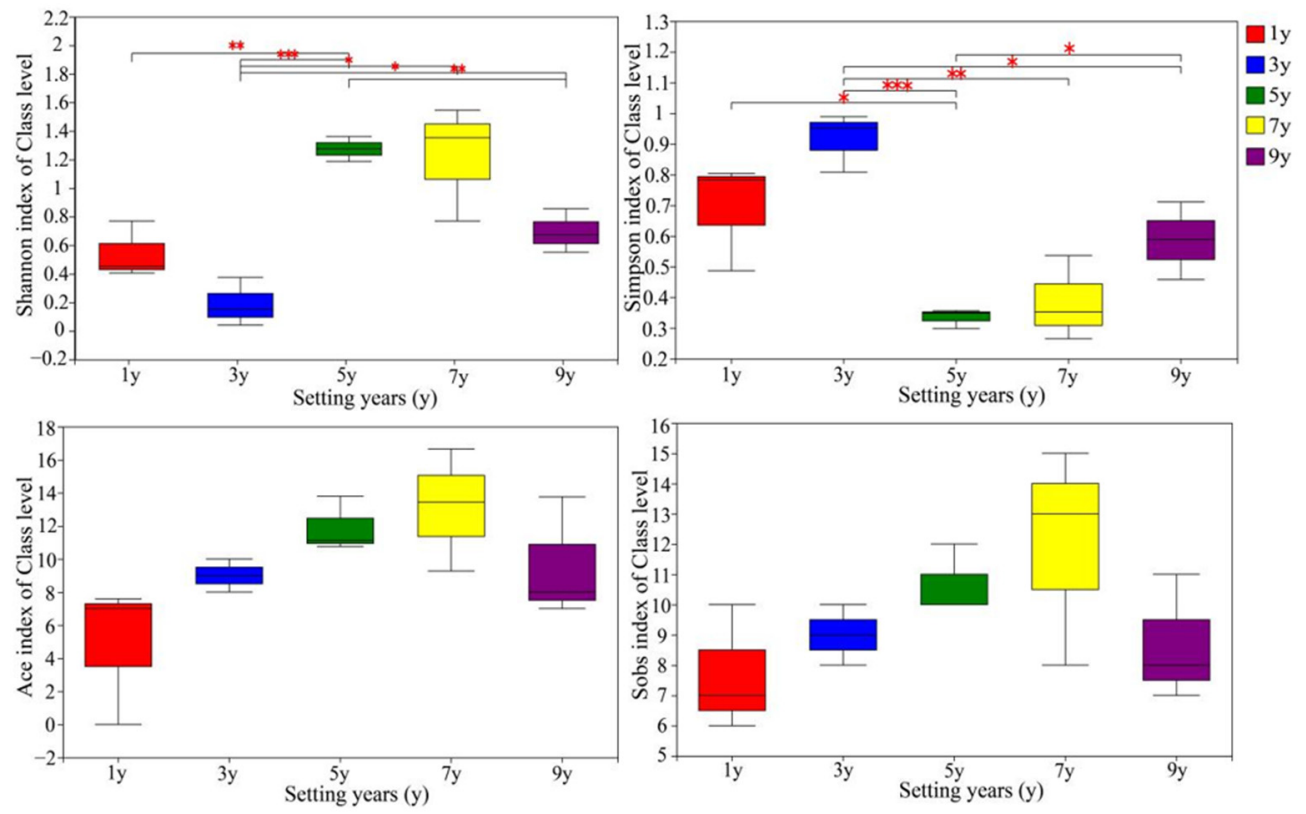

Figure 5. Box plot of inter-group differences in fungal community Alpha diversity indices. Note: $0.01<p \leq 0.05$ is marked as ${ }^{*} ; 0.001<p \leq 0.01$ is marked as ${ }^{* *}$; and $p \leq 0.001$ is marked as *** 


\subsection{Relationship between Fungal Community Structure and Environmental Factors}

As can be seen from Figure 6 (Supplementary Table S1), RDA1 explained $58.30 \%$ of the variation in the sample and RDA2 explained $21.30 \%$, together explaining $79.60 \%$ of the fungal community structure of $S$. psammophila sand barriers. As indicated in the RDA ordination diagram, the basic density, sand barrier sample moisture content, cellulose, and lignin were the main environmental factors affecting the distribution of the fungal community, while total $\mathrm{K}$ content had the least influence on the fungal community. The degree of influence of environmental factors on the samples is indicated according to the distance of the projection point from the origin. It can be seen that in the sand barriers, cellulose was the main microenvironmental factor influencing the structure of the fungal community in setting year one. Cellulose was also the microenvironmental factor that determined the community structure of the S. psammophila sand barrier fungus after setting for three years. Soil total $\mathrm{P}$ was the main factor determining the community structure of the S. psammophila sand barrier fungus after setting for five years. Moisture content of the sand barrier samples was the main contributor to the fungal community structure of the S. psammophila sand barriers after setting for five years. The $\mathrm{pH}$ was the main microenvironmental factor affecting the community structure of the S. psammophila sand barrier fungus. According to the angle between the arrows of environmental factors, the available $\mathrm{P}$, alkali-hydrolytic $\mathrm{N}, \mathrm{pH}$, dry-shrinkage rate, and moisture content of sand barrier samples had synergistic effects on the distribution of the S. psammophila sand barrier fungus community. The effects of total K, MOR, lignin, cellulose, basic density, hemicellulose, and soil moisture content on the fungal community were consistent. The angle between available soil $\mathrm{K}$ and total $\mathrm{P}$ was close to a right angle, indicating that there was almost no correlation between them. However, there was a negative correlation between available soil $\mathrm{K}$ and P. Based on the significance test results of the two ordering axes of each environmental factor, lignin, basic density, and volume shrinkage were significantly correlated with the two ordering axes $(p<0.01)$, and the correlation coefficients $r^{2}$ were $0.5483,0.5768$ and 0.6553 , respectively (Supplementary Table S1). Cellulose, hemicellulose, moisture content of barrier, bending strength, and soil total potassium were significantly correlated $(p<0.05)$ (Supplementary Table S1).

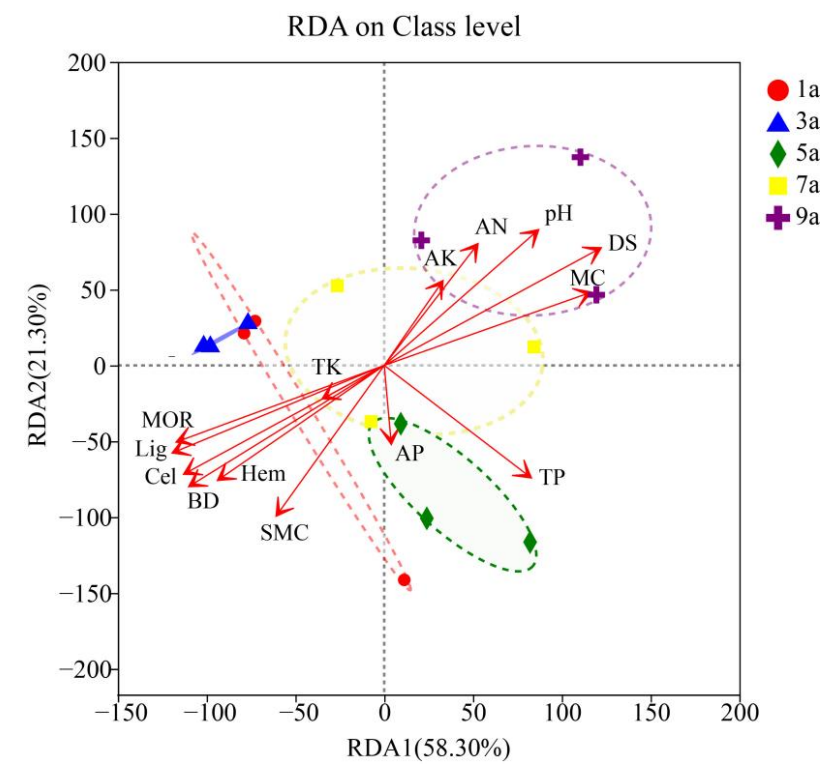

Figure 6. RDA analysis of fungal community structure and microenvironmental factors at different years. Notes: Lig, Lignin; Cel, cellulose; Hem, hemicellulose; BD, basic density; SMC, soil moisture content; MOR, modulus of rupture; TK, total potassium; TP, total phosphorus; AP, available phosphorus; AK, available potassium; AN, soil alkali-hydrolyzable nitrogen; DS, volume dry shrinkage; $\mathrm{MC}$, moisture content of sand barrier sample. 


\section{Discussion}

\subsection{The Decay Process of the S. psammophila Sand Barrier}

As expected, it is considered that setting for five years and seven years were inflection points for measuring the degree of decay of S. psammophila sand barriers; this result supported our first hypothesis. After setting for five years, the loss of physical parameters such as basic density, sand barrier sample moisture content, and shrinkage rate was less than $45 \%$, while the loss of mechanical properties was about $63 \%$ in terms of the MOR. After setting for seven years, the MOR decreased by $77 \%$. Some studies have similar results, showing that the lodging damage rate of $S$. psammophila sand barriers reached $80 \%$ after five years of installation, and that the wind-prevention and sand-fixation functions basically disappeared $[4,22]$. Our study further confirmed that, when the setting time is more than 7 years, change is relatively slow and tends to be stable. As the content of the main chemical components of the S. psammophila sand barrier decreased and reached an inflection point, the degradation products disappeared and the fungal activities were restricted. In the desert environment, with increased setting years, the physical and mechanical properties of the S. psammophila sand barriers are affected to different extents. Degradation of cell wall material results in decreases in physical properties such as barrier mass, basic density, and shrinkage rate $[23,24]$. The occurrence of dry shrinkage will not only change the original size and shape of natural biomaterials, but it will also cause defects such as decay due to the internal stress caused by uneven dry shrinkage, which will seriously affect the resource utilization of the S. psammophila sand barrier $[25,26]$. During this period, considering that the degradation products of cellulose and hemicellulose in the S. psammophila sand barrier gradually disappeared, the moisture content of the sand barrier showed an increasing trend, indicating that a certain relationship existed between the shrinkage rate of the sand barrier and its density, which was caused by the loss of water and chemical composition [26].

As the stable buried part of S. psammophila sand barriers is buried in the sand environment for a long time, cellulase and hemicellulase will be produced with the growth of microorganisms. Under the action of these enzymes, the cellulose and hemicellulose in the S. psammophila sand barrier will be decayed $[27,28]$. The degree of decay of the sand barrier differs due to the different rates of decomposition of different materials. The degradation rate of lignin is slower than that of cellulose and hemicellulose. The complex chemical structure of lignin has been previously elucidated [29]. There are three monomers of lignin: $p$-hydroxyphenyl propane, guaiacyl propane, and syringyl propane. Lignin is formed by the disordered combination of these monomers through dehydrogenation polymerization and $\mathrm{C}-\mathrm{C}$ bonding and $\mathrm{C}-\mathrm{O}$ bonding. The complex structure of lignin leads to the complex chemical reaction of lignin $[30,31]$. The stable buried part of S. psammophila sand barriers avoids direct ultraviolet rays and easily absorbs precipitation from the atmospheric environment or the air-condensed water formed by strong temperature differences, thus creating an excellent environment suitable for the growth of fungal communities [2]. Different fungi can produce a variety of lignocellulosic complex enzymes which decompose lignin and cellulose, resulting in the decay and degradation of the cell wall of the S. psammophila sand barrier [31,32]. The results showed that the contents of cellulose, hemicellulose, and lignin in the sand barriers decreased with the increase in setting years. The content of cellulose and hemicellulose decreased significantly after setting for one to seven years, and the change was relatively gentle after setting for seven years. Among them, the loss of lignin content was relatively small and showed a general decreasing trend, which might be because the cellulose and hemicellulose composed of polysaccharides were more easily decomposed, while only a small amount of lignin was decomposed.

\subsection{Analysis of Factors Influencing the Fungal Community Structure}

Sordariomycetes and Eurotiomycetes were the main fungal groups in the process of S. psammophila sand barrier decay, and both exist widely in nature. Sordariomycetes accounted for the highest proportion among different years, including many important plant pathogens and saprophytic fungi [33]. Eurotiomycetes fungi are the most ecologically di- 
verse fungi that can decompose the organic residues of plants, thus playing an important role in the carbon cycle of the ecosystem [34]. The species abundance of Eurotiomycetes increased significantly after setting for five years, accounting for the highest proportion (up to 51.87\%) after setting for nine years. With the change in the degree of decay, the richness of the fungal community of $S$. psammophila sand barriers first increased and then decreased. The richness of the fungal community of the sand barriers reached its minimum after setting for one year and reached its maximum after setting for seven years. This result differs slightly from the results of Makipaa et al. [35], which may be due to the fact that the selected barrier samples were set up for a long time, showing the time inflection point of decline of the fungal community richness. After setting for seven years, the utilization of cellulose, hemicellulose, and other major chemical components in the fungal community decreased, and it was difficult for the fungi to maintain normal rapid growth and reproduction, leading to the decrease in the number of fungal species and the decline of community richness.

The RDA analysis showed that the differences in various microenvironmental factors, such as the physical and chemical properties of the surrounding soil, affected the changes in fungal community structure and quantity. Among them, lignin, cellulose, basic density, and sand barrier moisture content had a great influence on the fungal community distribution. Lignin and cellulose, as $\mathrm{C}$ and $\mathrm{N}$ sources for fungal growth, determine the variation in fungal species and number [31]. As the main component of fungal mycelium, the water in the $S$. psammophila sand barrier also contains the complex enzymes secreted by fungal cell wall decomposition, which directly affect the germination of fungal spores and the growth of fungal mycelium [31,36]. This article revealed the relationship between fungal community distribution and microenvironmental factors in the process of S. psammophila sand barrier decay. The relationship between fungal community and the decay process of the sand barrier still needs further studies.

\section{Conclusions}

1. With increased setting years, the physical and mechanical properties of the S. psammophila sand barriers degenerated to varying degrees, and the main chemical components were lost. After five years of setting, the physical parameters of basic density and shrinkage rate decreased by $44.04 \%$ and $28.68 \%$, respectively, while the loss of the MOR mechanical index was $62.72 \%$. After setting for seven years, the physical parameters of basic density and shrinkage rate declined by $62.70 \%$ and $43.93 \%$, respectively, whereas the MOR decreased by $76.95 \%$. Setting for five and seven years were important inflection points for measuring the decay process of the sand barriers.

2. Sordariomycetes (53.75\%) and Eurotiomycetes (19.78\%) were the main fungi groups associated with the decay of $S$. psammophila sand barriers. Leotiomycetes was the most abundant species, accounting for $21.93 \%$ after five years. Fungal community richness first increased and then decreased, and the greatest community richness was detected after seven years. Basic density, sand barrier moisture content, cellulose, and lignin were the main environmental factors affecting the distribution of fungal community in the sand barriers.

Supplementary Materials: The following are available online at https:/ / www.mdpi.com/article/10 .3390 / su13147590/s1, Table S1: Correlation of microenvironmental factors with the RDA of fungal communities in the sand buried part of Salix psammophila sand barriers.

Author Contributions: Writing—original draft, X.Y. and Y.L.; resources, Y.G.; Writing-Methodology, Original draft preparation, R.W. All authors have read and agreed to the published version of the manuscript.

Funding: The research was supported by the National Natural Science Foundation of China (41861044), "The enhancement mechanism of atmospheric-sand dynamic process on Salix sand barrier resistance."

Institutional Review Board Statement: Not applicable. 
Informed Consent Statement: Not applicable.

Data Availability Statement: Not applicable.

Conflicts of Interest: The authors declare that they do not have any conflict of interest.

\section{References}

1. Gao, Y.; Gong, P. Salix Sand Barrier; Beijing Science Press: Beijing, China, 2013.

2. Wang, R.D.; Dang, X.H.; Gao, Y.; Yang, X.; Liang, Y.M.; Qi, S. Alternated desorption-absorption accelerated aging of salix psammophila sand barrier. Bioresources 2020, 15, 6696-6713. [CrossRef]

3. Gao, Y.; Yi, Y. Studies on suitable vegetational cover rate for Salix psammophila forest. Inn. Mong. For. Sci. Technol. 1996, 3, 38-42.

4. Gong, P. The Corrosion Processes of Salix Sand-Barrier and the Effect of Anticorrosion. Ph.D. Thesis, Inner Mongolia Agricultural University, Hohhot, China, 2012.

5. Wang, L.Y.; Li, H.L.; Dong, Z.; Wang, L.Q.; Liu, F.C. Effect of Salix psammophila checkboard sand barrier on wind prevention and sand resistance in Kubuqi desert. J. Soil Water Conserv. 2013, 27, 115-124.

6. Pointing, S.B.; Belnap, J. Microbial colonization and controls in dryland systems. Nat. Rev. Microbiol. 2012, 10, 551-562. [CrossRef] [PubMed]

7. Murgia, M.; Fiamma, M.; Barac, A.; Deligios, M.; Mazzarello, V.; Paglietti, B.; Cappuccinelli, P.; Al-Qahtani, A.; Squartini, A.; Rubino, S.; et al. Biodiversity of fungi in hot desert sands. MicrobiologyOpen 2019, 8, 1-10. [CrossRef]

8. Carlsson, F.; Edman, M.; Jonsson, B.G. Increased $\mathrm{CO}_{2}$ evolution caused by heat treatment in wood-decaying fungi. Mycol. Prog. 2017, 16, 513-519. [CrossRef]

9. Varela, A.; Martins, C.; Núñez, O.; Martins, I.; Houbraken, J.A.; Martins, T.M.; Leitão, M.C.; McLellan, I.; Vetter, W.; Galceran, M.T.; et al. Understanding fungal functional biodiversity during the mitigation of environmentally dispersed pentachlorophenol in cork oak forest soils. Environ. Microbiol. 2015, 17, 2922-2934. [CrossRef]

10. Pouska, V.; Macek, P.; Zíbarová, L.; Ostrow, H. How does the richness of wood-decaying fungi relate to wood microclimate. Fungal Ecol. 2017, 27, 178-181. [CrossRef]

11. Küffer, N.; Senn-Irlet, B. Influence of forest management on the species richness and composition of wood-inhabiting basidiomycetes in Swiss forests. Biodivers. Conserv. 2005, 14, 2419-2435. [CrossRef]

12. Brazee, N.J.; Lindner, D.L.; D'Amato, A.W.; Fraver, S.; Forrester, J.A.; Mladenoff, D.J. Disturbance and diversity of wood-inhabiting fungi: Effects of canopy gaps and downed woody debris. Biodivers. Conserv. 2014, 23, 2155-2172. [CrossRef]

13. LY/T 2369. Testing Methods for Physical and Mechanical Properties of Desert Shrub; Standardization Forestry Industry of China: Beijing, China, 2014.

14. Wang, L.F.; Cheng, Y.C. Determination the content of cellulose by nitric acid-ethanol method. Chem. Res. 2011, $22,52-55$.

15. Xiong, S.M.; Zuo, X.F.; Zhu, Y.Y. Determination of cellulose, hemi-cellulose and ligin in rice hull. Cereal Feed Ind. $2005,8,40-41$.

16. Zhang, B.P.; Yang, F.; Ma, Z.C.; Guo, M.C.; Liu, Y. Extraction and characterization of the lignin from rice straw by sulfuric acid. J. Hebei Univ. (Nat. Sci. Ed.) 2016, 36, 474.

17. BAO, S.D. Analysis of Soil Agrochemical, 3rd ed.; China Agriculture Press: Beijing, China, 2000.

18. Nilsson, R.H.; Larsson, K.H.; Taylor, A.F.S.; Bengtsson-Palme, J.J.; Thomas, S.; Schigel, D.; Kennedy, P.; Picard, K.; Glöckner, F.O.; Tedersoo, L.; et al. The UNITE database for molecular identification of fungi: Handling dark taxa and parallel taxonomic classifications. Nucleic Acids Res. 2019, 47, 259-264. [CrossRef] [PubMed]

19. Schoch, C.L.; Seifert, K.A.; Huhndorf, S.; Robert, V.; Spouge, J.L.; Levesque, C.A.; Chen, W.; Bolchacova, E.; Voigt, K.; Crous, P.W. Nuclear ribosomal internal transcribed spacer (ITS) region as a universal DNA barcode marker for Fungi. Proc. Natl. Acad. Sci. USA 2012, 109, 6241-6246. [CrossRef]

20. Zhang, S.Y. Wood specific gravity-mechanical property relationship at species leve. Wood Sci. Technol. 1997, 31, 181-191. [CrossRef]

21. Feng, L.Q.; Gao, X.X.; Wang, X.M. Microstructure and chemical composition analysis of Salix psammophila. J. Inn. Mong. For. Univ. 1996, 18, 38-42.

22. Wang, R.D.; Dang, X.H.; Gao, Y.; Yang, X.; Zhang, C.; Li, W.J. Damage Law and Vegetation Restoration of Salix psammophila Sand Barrier in Mu Us. J. Southwest For. Univ. 2019, 39, 71-77.

23. Wang, R.D.; Gao, Y.; Dang, X.H.; Yang, X.; Liang, Y.M.; Zhao, C. Microstructure and biodegradation of long-established Salix psammophila sand barriers on sand dunes. Environ. Technol. Innov. 2020, 21, 101366. [CrossRef]

24. Chi, Y.J. Wood Decay and Wood Decay Fungi; Science Press: Beijing, China, 2003.

25. Yang, T.T.; Ma, E.N. Dynamic sorption and hygroexpansion of wood by humidity cyclically changing effect. J. Funct. Mater. 2013, $44,3576-3580$.

26. Ma, E.N.; Zhao, G.J. Hygroexpansion of wood: From equilibrious state to non-equilibrious state. J. Beijing For. Univ. 2006, 28, 133-138.

27. Beguin, P. Molecular biology of cellulose degradation. Annu. Rev. Microbiol. 1990, 44, 219-248. [CrossRef] [PubMed]

28. Ljungdahl, L.G.; Eriksson, K.E. Ecology of Microbial Cellulose Degradation. In Advances in Microbial Ecology; Springer: Boston, MA, USA, 1985; pp. 237-299.

29. Zimmerman, W. Degradation of lignin by bacteria. J. Biotechnol. 1990, 13, 119-130. [CrossRef] 
30. Wang, Y.L. Study on C-C Bond Cleavage of Lignin and Models via BaeyerVilliger Oxidation and Beckmann Rearrangement and Photoinduced by Ircomplex and $\mathrm{CeCl}_{3}$. Ph.D. Thesis, Jilin University, Changchun, China, 2019.

31. Wu, X.F.; Su, X.J.; Chen, L.; Chen, J.P.; Wang, K.Q. Research progress in analyzing methods of lignin structure. Renew. Energy Resour. 2015, 33, 267-274.

32. Nait M'Barek, H.; Arif, S.; Taidi, B.; Hajjaj, H. Consolidated bioethanol production from olive mill waste: Wood-decay fungi from central Morocco as promising decomposition and fermentation biocatalysts. Biotechnol. Rep. 2020, 28, e00541. [CrossRef] [PubMed]

33. De Hoog, G.S.; Queiroz-Telles, F.; Haase, G.; Fernandez-Zeppenfeldt, G.; Attili Angelis, D.; Gerrits Van Den Ende, A.H.; Matos, T.; Peltroche-Llacsahuanga, H.; Pizzirani-Kleiner, A.A.; Rainer, J.; et al. Black fungi: Clinical and pathogenic approaches. Med. Mycol. 2000, 38, 243-250. [CrossRef] [PubMed]

34. Kirk, P.M.; Cannon, P.F.; Minter, D.W. Ainsworth and Bisby's Dictionary of the Fungi, 10th ed.; CAB International: Wallingford, UK, 2008.

35. Mäkipää, R.; Rajala, T.; Schigel, D.; Rinne, K.T.; Pennanen, T.; Abrego, N.; Ovaskainen, O. Interactions between soil and dead wood-inhabiting fungal communities during the decay of Norway spruce logs. ISME J. 2017, 11, 1964-1974. [CrossRef]

36. Chen, K.Y.; Qiu, H.X.; Zhu, Z.M. Bearing capacity of timber beams with shrinkage cracks. J. Civ. Archit. Environ. Eng. 2018, 40, 39-47. 